\title{
Mobile Technology based Polio-Vaccination System (PVS) - First Step Towards Polio-Free Pakistan
}

\author{
Nukhba Afzal \\ Dept. of Computer Science and IT \\ The Islamia Univ. of Bahawalpur, \\ Pakistan
}

\begin{abstract}
Malik Muhammad Saad Missen
Dept. of Computer Science and IT

The Islamia Univ. of Bahawalpur, Pakistan
\end{abstract}

\author{
Amnah Firdous \\ Dept. of Computer Science \\ CIIT, \\ Vehari
}

\author{
Nadeem Akhtar \\ Dept. of Computer Science and IT \\ The Islamia Univ. of Bahawalpur, \\ Pakistan
}

\author{
Hina Asmat \\ Dept. of Computer Science and IT \\ The Islamia Univ. of Bahawalpur, \\ Pakistan
}

\author{
Saleem Ullah \\ Dept. of Information Tech \\ KFUEIT, \\ Rahimyar Khan
}

\begin{abstract}
Health information technology revolutionized the world with its great expansion and widespread in the domain of health care system. Most of the developed countries adopted advanced technology in their vaccination systems. Vaccination systems of many developing countries still lack the use of technology eventually causing mismanagement and corruption to occur in vaccination campaigns. Issues like mismanagement and corruption not only affect vaccination campaigns but also cause further diffusion of a disease. Pakistan is also one of such countries where vaccination system is prone to these and many other issues and hence it does not help in disease eradication. For example, polio remains alive in Pakistan because Pakistan's Polio vaccination system is faced with many problems and the biggest one is security of vaccination teams. Corruption, mismanagement, unawareness among public and life-threat to vaccination teams are the main problems of current polio vaccination system of Pakistan. To overcome these flaws and to make an idyllic system with the new advanced technology, we propose technology oriented secure polio vaccination system. The proposed system is more secure and removes flaws in the current system. We model our proposed system using Colored Petri Nets (CPNs) which is a state-of-the-art tool for formal modeling.
\end{abstract}

Keywords-Polio Vaccination; Information system; GPS technology; Health-care

\section{INTRODUCTION}

Advancement in technologies has led to the development of health care technologies. Technology in health care encourages the people to adopt healthy life style along with the advancement of technology-based interventions made to improve the working of health-care like remotely access the doctors, , clinical management support, supporting clinical diagnosis and treatment, send patients diagnosed results on time through SMS based appointment reminders, timely response. Thus there is a need to adapt technology for healthcare system especially for under-developed countries and especially for the infectious diseases. Polio is one of the infection disease caused by poliovirus and still spreads only in Pakistan, Afghanistan and Nigeria [1, 2, 3]. Existing polio vaccination system of Pakistan is incompetent and ineffective. Mismanagement, corruption and insecurity are major problems of the existing system. These lacking take the vaccination campaign towards the termination and increase the rate of missed children. Therefore, there is a need of such a system which can cope with current challenges and have a capability to cope up with the current time and technology.

Polio remains endemic in two countries of the world Afghanistan as well as Pakistan. Polio endemic countries could be the reason of importing wild polio virus to those countries that are non-polio endemic [5].Some measures have been taken to stop this spread of polio virus to other nations like some countries who are not endemic already declared polio vaccination mandatory for endemic countries travelers, who are not immunized against polio. Besides this, health care providers have identified the technologies with different purposes like in educating the people, diagnosing the diseases; management and communication between patient and health service providers to further cope with this infectious disease. However, immunization is considered to be one of the greatest health interventions to prevent polio. There is a need of making the process of immunization more effective by leveraging the support of advance technology. Existing health care systems with integration of new mobile technology can help to eradicate polio from world map $[4,6,7]$.

In Pakistan, polio immunization campaigns have been facing nonstop setbacks. According to 2015 report of World Health Organization (WHO), 51 confirmed cases of polio virus occurred in Pakistan. There are many reasons behind having Polio in Pakistan that include lack of education and awareness, religious concerns, mismanagement, corruption and life threats to vaccinators.

In this paper, we propose a very effective technology based polio vaccination system for Pakistan after identifying the drawbacks in the current system. The main objective is to make the polio vaccination system efficient in general and security perspective. Without proper security of health 
workers and general public it is not possible to carry out a successful campaign [3] $]^{1}$.

\section{A. Research Objectives}

Our aim is to rectify the current polio vaccination system of Pakistan by making an effective use of technologies:

- To perform the detail study of current polio vaccination system of Pakistan to identify the problems and flaws (general as well as security related) in the current polio vaccination system,

- To propose a new technology based and secure polio vaccination system,

- To formally model our proposed system using formal modeling techniques.

\section{RELATED WORK}

We discuss related work with respect to countries that have their polio vaccination working.

\section{A. United States}

Technology is widespread in US with its great expansion. This extensive and prevalent usage of advanced tools gives an idyllic and perfect platform in order to help in the deliverance of vaccines.

The presence of electronic health record utilizing and the resultant capability to have vaccination data in E-record form grant an imperative establishment for delivering IT based vaccine interventions. Vaccination system become rationalized with the advanced tools like use of E-data, increase the number of patients interacted and contacted with the intervention $[3,8]$.

In United States, National vaccine recommendations goal is to increase the amount of vaccine-preventable diseases for diminution, abolition, or abolition. One of their objectives is to check out the rate of vaccination before the implementation of suggested vaccination and its preventable diseases with the comparison of transience and death rate $[8,9]$.

Electronic data made the work easier in keeping the accurate and reliable data. Whenever data is needed, there is no need to check out or find out the manual data like people do before the advancement of the advanced tools and technology. Computerized registries are being done now days, which help to sustain the record with its confidential. Once electronic record has been created, it can be used repeatedly or can be used by any other organizations as well like public organizations or any semi government organizations. Now a day in health care system, management can easily maintain the record of infants or adults. Members of health provider could easily fetch out the data of patient anytime or anywhere and its relevant record [3].

\section{B. Nigeria}

Nigeria has made extraordinary growth against wild poliovirus. World Health Organization (WHO) publicized that polio virus is not any more in Nigeria in September 2015.No case of wild poliovirus has reported in Nigeria from 2014. For the very first instance, Nigeria has broken up importation of wild polio virus, taking the nation state and the entire African territory nearer than ever to being experienced as free polio virus in the world.

Recently in 2012, most of the polio virus cases reported in Nigeria globally. Ever since, an intensive attempt by the management of Nigeria, religious leaders, civil society and thousands of enthusiastic vaccinators of polio virus have resulted in Nigeria productively bring it to halt. Not only had this but also improving vaccination coverage and adoption of new advanced technology in vaccination coverage also helped in reaching the goal.

Nigeria changed their way of primitive coverage of vaccination by adopted advanced tactics. They identified the programmatic weakness in polio campaign, tried to improve training, planning, supervision and accountability. They worked on global positioning system (GPS) device to improve the coverage and to easily track the vaccination teams.

Currently, tracking of different teams of vaccinators with GPS is carried of each campaign wise. A device is given to each team of vaccinator before the initiation of their work. As they finished their per day work assignment they again give a call to the head quarter about the finishing of their daily bases job. After taking the devices back from the each team of vaccinators, they call up for the daily progress report and reviews. So, this workflow is followed till the accomplished of vaccination process [9].

Such a project with Graphical information system technology improved the quality of micro planning and provided information about team performance. It also provided tools in identified missed children. Different branch to the Geographical Information System toil in Nigeria:

1) To targeting the different states of Nigeria with the GPS based digital map, it gathers the data with its relative boundary.

2) Incorporating of digital the map into the micro planning procedure,

3) A group of people has been bounded to keep eyes on the vaccinator along with their teams, with GPS tracker to chase them and to reach them with the help pg GPS navigation or the digital map.

4) Giving timely based reviews and feedback.

5) A web based portal has been set in with the GPS tracking system to view working of vaccination teams. So

\footnotetext{
${ }^{1}$ Poliomyelitis Fact sheet N$^{\circ} 114 "$. WHO int. October 2014. Retrieved 3 November 2014
} 
management can check out the way campaign conducted at local level or remote level [9].

The use of digital map instead of old hand drawn map improves the campaign. Before that, hand sketched map were used, which were often incomplete and inaccurate. Small region or hamlet areas missed in hand drawn map and only major cities were located. Hamlet areas are more crucial in providing immunization. Identification of the village areas is tricky, while they are an artificial erect based on immediacy. Digital maps are printed for each and every zone for use in micro planning. An outline is used for the micro plan, together with the maps, is also made accessible via web based portals. The maps and the given outline is used by the each zone team. According to the area or the population, these essentials are given to each vaccination teams till the completion of vaccination campaign.

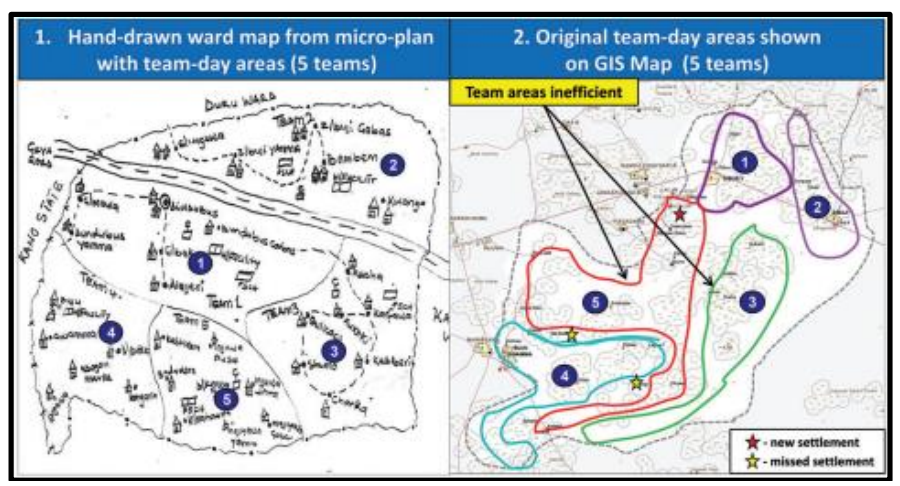

Fig. 1. Human hand drawn and geographical system maps

Nigeria did their best to completely eradicate the polio virus from their country. They used the new advanced technology and other tools which help them out in stopping polio virus from their territory. Nigeria worked very efficiently and in a technical manner in achieving their goal, now they proved them self by eradicating the polio intelligently. All this can be done by the hard work of their government, their strategies and policies as well vaccinators and involvement of many brilliant minds behind it.

\section{India}

India eradicated the wild virus from its territory and claimed its eradication of polio around the globe. In India, they worked hard in disrupting the importation of wild polio virus from their region and sustained this interruption for 2 years to declaring its polio free region. They checked the infant and their adults and confirmed the negative reports in their all territories. They examined their water or wastage system on weekly basis and verified all the samples and got negative results. Hence, in 2012 "WHO" detached the India from endemic countries of the world.

When no chances of transmission occur in their region indicated the confirmation of polio virus. After complete examined the India after 2011, a certification of polio free countries is given. In the start, India used OPV vaccine instead of IPV. By using OPV, it will only weaken the polio virus germ but germs still remain in the body of effected one. Then they started the use of IPV, inactivated polio vaccine, they inactivated the wild polio germs in the body of patient and gradually all the germs becomes inactivated and destroyed. IPV did a remarkable achievement in the negative polio results. India most effected region was Bihar and Uttar Pradesh where mostly cases of wild polio virus in infant and adult was reported. Government of Bihar and Uttar Pradesh did their best in disrupting the polio virus. Finally, they achieved their target in disrupting the polio virus in their areas. During that time, they conducted more than ten times of vaccination campaign in a year to determine the status of conformation of polio virus and worked according to the outcomes of their coverage. Thus in their $2^{\text {nd }}$ phase of eradication of polio virus cases, they scrutinized each and every infant of their respective regions and did their best in interrupting the wild polio virus.

The entire struggle behind the polio virus campaigns, they cleared their region with $0 \%$ of polio virus with IPV injected vaccine and hard work of day and night. True polio eradication required $0 \%$ chances of polio virus. India did this and proved their region completely polio free in the world [1, $10]$.

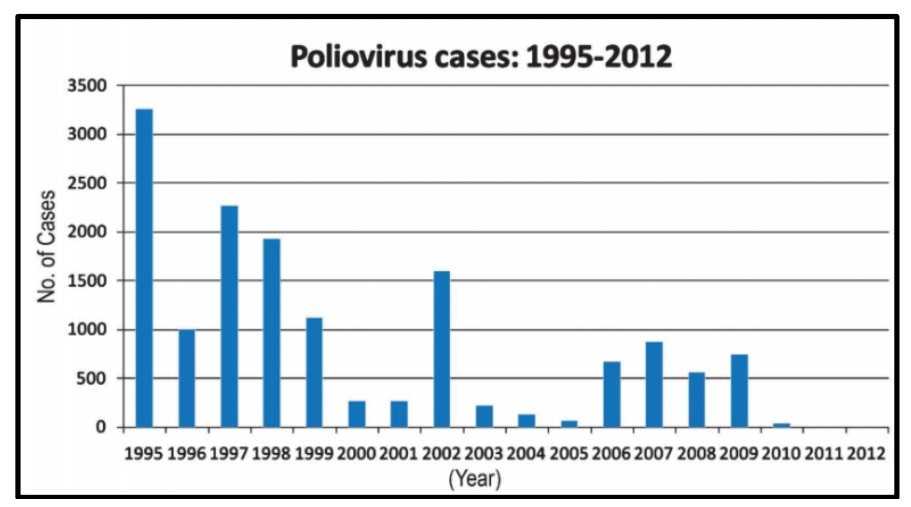

Fig. 2. Occurrence Wild polio cases in India

Finally, after the long struggling journey with polio India succeeded and declared as a polio free country. In last 3 years not even a single case reported from anywhere throughout the country regarding the wild poliovirus. Last case of poliovirus was reported from West Bengal State of India and the nature of the case was polio virus in January. Unfortunately polio virus has not been identified in sewage test in that span of time. India still needs to sustain his position for next 2 years to fully declare as polio free country. The main circumstances in India which makes the reason of polio cases had been the rejection of polio vaccine by few communities due to lack of education and propaganda against polio virus. On the other hand, regime of India playing a vital role by engaging/taking other stake holders on board which include media as well. Government of India also employed 2.5 million plus health workers who worked days and nights and had successfully achieved this landmark $[11,12]$.

\section{SURVEYS}

The primary goal of these surveys is to identify the problems in current polio vaccination system. The target populations of surveys are general public and vaccination teams. Another objective of these surveys is to find all those factors, policies, practices and reasons causing unsuccessful 
campaign and unsafe vaccination process and having polio virus in Pakistan.

We make sure that the survey queries are modified according to the specific groupings i.e. we ask non-technical questions from non-technical population like general public and similarly we ask technical questions from technical people like vaccinators or health care provider. These two types of target populations, we design two surveys with different questions in each questionnaire.

\section{A. General Public Survey}

In general public questionnaire, there are almost 51 questions which were asked from the parents of immunized child in last 6 months to 1 year. Hand written questionnaire was given to each person. This questionnaire is in-depth study of Polio vaccination system and its services. This report focus solely on the findings related to the vaccination system. Questionnaire results will serve to urge the presence of faults and lacking in the system where the further improvement can be made.

\section{Survey Findings}

After analyzed the survey data, we organized the data and highlights the important findings from general public survey, is as follows

- Polio is still the issue of Pakistan and its vaccination is necessary.

- Parents need more information to vaccinate their child.

- Parents missed the vaccination of their child mostly.

- Missed child could not be contacted for vaccination.

- Polio Vaccination system is older than current time and advancement of technology

- People spread wrong information and gives ill-advice to others.

- Polio Vaccination system is not safe and need to make the system safe for Polio workers.

- We have Polio in Pakistan because of insecurity.

- There should be more focus on danger zone areas.

- It is the need of time to make the system efficient and competent with respect to security and management

Parents don't believe in the use of whether they use either from philosophical aspects reasons or as a consequence of religious concerns and beliefs, and similar proportion of such a people has concerns about the safety of usage of vaccines

\section{B. Vaccination Team Survey}

In vaccination team survey, there are almost 65 questions which were asked from the vaccinators and health workers. According to the survey, ten out of ten strongly believe that Polio is public health problem in Pakistan and all the children should be given Polio vaccine. Only three in ten attended/heard about Polio vaccination campaign. $100 \%$ vaccinators strongly believe that Vaccination eradication campaigns should be carried out. Seven out of ten strongly agree while 2 are agreeing on that there is still need to focus more on the activities towards Polio awareness campaign.

\section{Survey Findings}

After analyzed the survey data, we organized the data and highlights the important findings from general public survey, is as follows:

- Polio is still the issue of Pakistan and vaccination should be necessary and must be applicable in Pakistan.

- Vaccination eradication campaign needs to work more.

- There is still need to enhance the Polio awareness campaign.

- Vaccination system is older than current time and advancement of technology.

- Campaign need to be improved operationally and its management should be enhanced.

- Complaints of health workers are usually occur.

- No record of immunization found electronically

- Vaccination system does not have any strategy to tracked missed children.

- Vaccination has so many lacking on the subject of security, operations and communication

- Vaccination system is not secure and need to make the system safe for Polio workers.

- Danger zone areas need more attention and focus

- There is need to make the system efficient and competent with respect to the security and management

- Poor Security is the subject of matter of having Polio in Pakistan.

- Polio vaccination systems of Pakistan have not any proper technique to cover up those areas which are exposed to high risk.

- High risky areas are being neglected because of poor security and administration is unable to pay attention on danger zone areas.

- There is need to improve the way of campaign being conducted in risky areas

- Insecure vaccination system ruins the whole campaign and disheartens the workers.

- Security system requires improvement and enhancement.

- Poor security system is the reason of Polio cases in risky areas.

- People do not inject vaccine because of theoretical and religious mind set. 


\section{FLAWS IDENTIFIED IN CURRENT PVS OF PAKISTAN}

Followings flaws have been identified in current vaccination system after two full fledge surveys.

\section{A. No/incomplete immunization record}

Current polio vaccination system of Pakistan has a very poor record keeping process. Most of the time no record is maintained by the staff or fake entries is made. Even if staff maintains the record regularly, the data being kept is not enough to keep record of missed children or dual entries. Figure 3 and 4 are examples of current record keeping.

\section{B. Paper based vaccination system}

Vaccination records are completely maintained on papers. Child immunization registration is done on hand written paper. Paper based systems are prone to many human mistakes where there is no system of error feedback. Hence, the quality of data is uncertain and inaccurate. Figure 5 and 6 are examples of current record keeping.

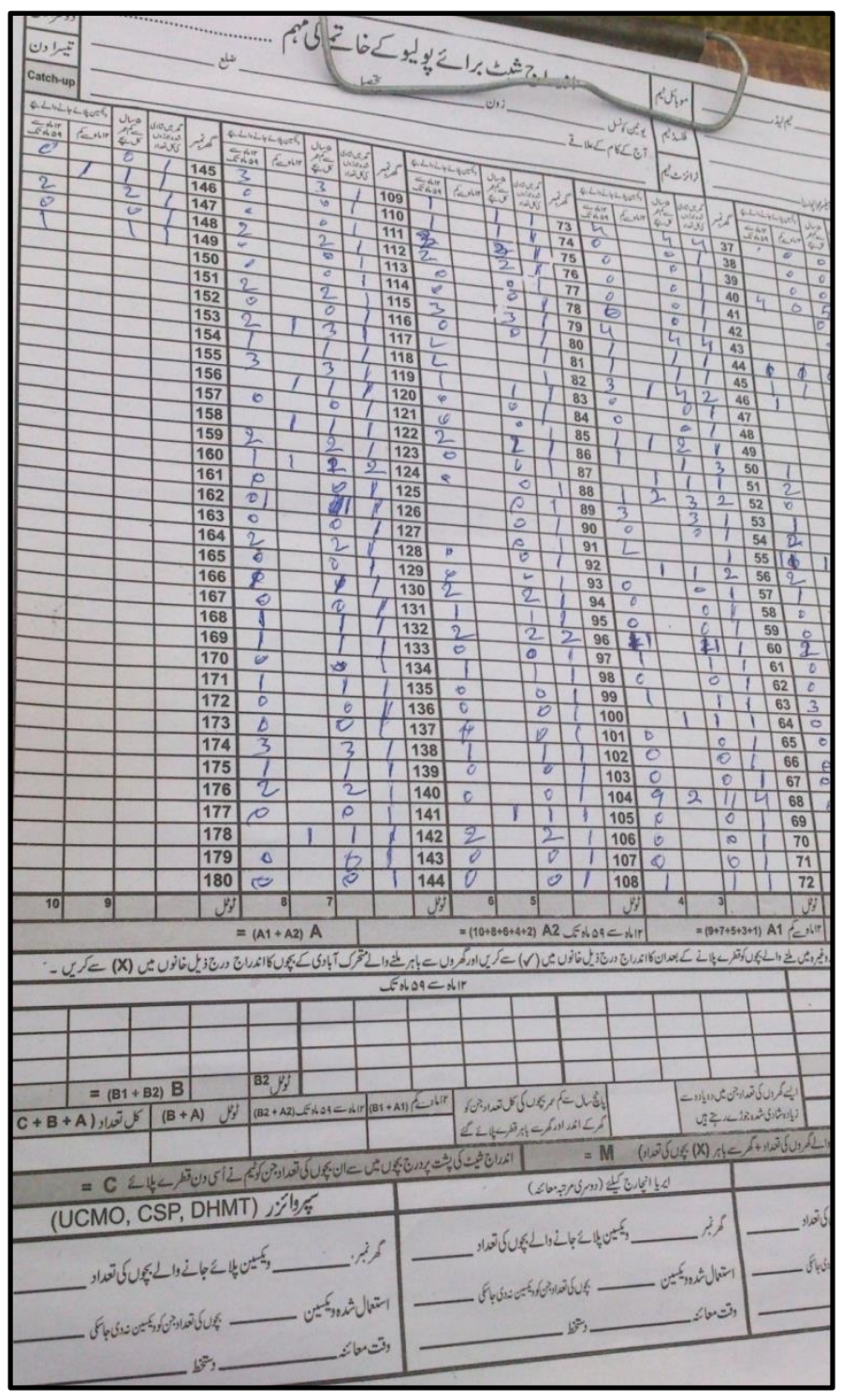

Fig. 3. Entry List for the Immunization Regarding Polio Eradication

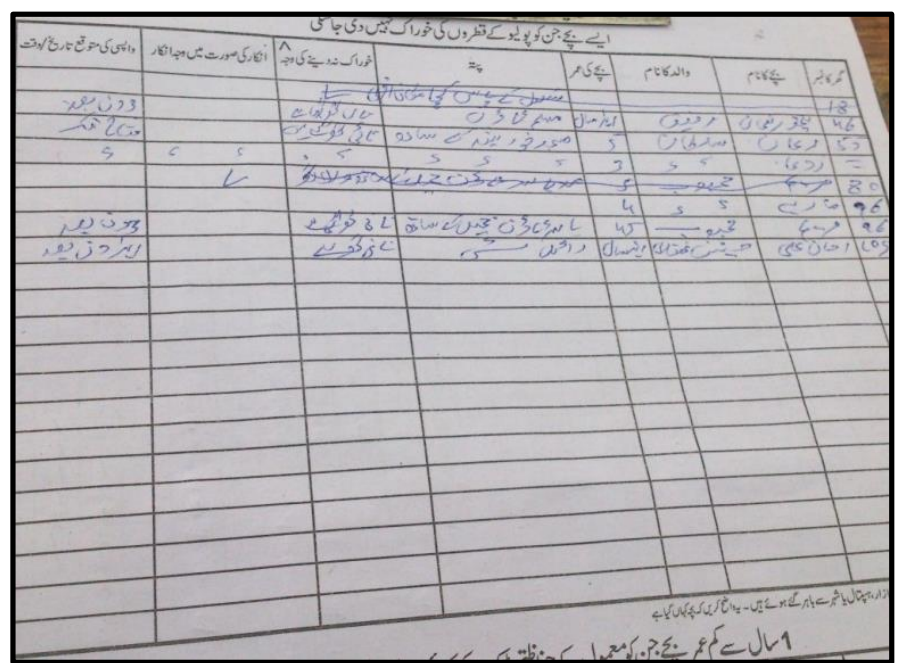

Fig. 4. Entry List for the Immunization Regarding Polio Eradication

\section{High rate of missed children}

During campaign, most of children remain deprived of vaccination or some are vaccinated twice. Poor record keeping and work corruption are main reasons of this problem. Unfortunately, there are no specific policies or laws being set which facilitate to immunize those missed children. This is the significant factor of rising polio cases in Pakistan.

\section{Lack of Technology}

In current vaccination process, lack of technology limits the capacity and effectiveness of the health worker. There is speedily widespread adoption of new technology in immunization system in developing countries. It is the need of the time to push down the new technology in the system. Use of advanced technology in vaccination system, it will not only be the well-organized structure but also a proficient system.

\section{E. Mistrustful information about Polio Campaigns}

Misinformation has destabilized polio vaccine campaigns in Pakistan. Misconceptions and lack of education are considered as foremost barricades regarding polio vaccination. Parents are unaware of the threats of vaccine preventable disease. People especially in remote areas have so many misconceptions about vaccines. They fully negate to immunize, for not having a proper awareness about vaccine and its benefits. Thus, incredible information and misconception increase the barrier in the delivery of immunization.

\section{F. No Security Zone in High Risk Areas}

High security risk areas have denied access to some part of the population and hinder the growth of 'End Polio from Pakistan' campaign. This is one of the major reasons behind the presence of polio cases in Pakistan. In Pakistan the main challenges in scheming polio conduction consist of poor and inappropriate security situations and unreachable areas with geological barricade. Infants are usually unapproachable and health care takers face intricacy in upholding polio virus campaigns productively. Threatening and killing polio health workers discourage them in performing their duties. This leads to halt on the vaccination campaign. Such Mishaps dispirit the 
community and they refuse vaccination too. In 2012 Pakistani Taliban commander banned the polio vaccination in Federally Administrated Tribal Areas (FATA) of Pakistan in reaction of United States drone attacks. Most of the polio cases are also found in FATA, Khyber Pakhtunkhwa (KPK), Baluchistan, Karachi and its surrounding areas. In Baluchistan, numerous cases were reported in from Quetta division. This growing complexity in vaccination process increases the need of high security measures. Security risks continue in Karachi and its related areas. Thus it is very difficult to maintain the quality of campaign and to continue with it. Most recently case was occur in April, 2016 in Karachi an attacked on polio team during vaccination campaign, killed cops which were on duty as a guards of polio team.

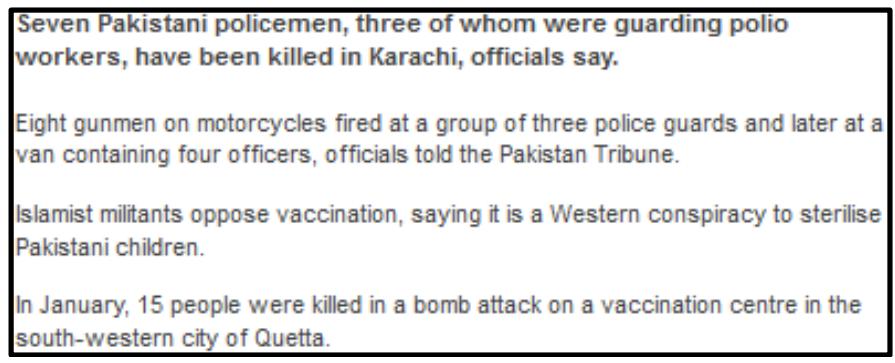

Fig. 5. Attack on the polio team in April 2016 [13]

\section{G. Poor performance of vaccinators}

The role of health service providers in the eradication of polio virus program can't be overlooked. The vaccinators already know their assigned regions or community, the territory and the language of the area in which they work, facilitating the job of governing the vaccine with a high rate of coverage. They can better immunize the children and increase the rate of immunization. The efforts of millions of people from different communities and walks of life have made anti poliovirus activities successful by performing their duties with punctuality, dedication and sincerity. Unfortunately, most of the vaccinators cannot carry out their task properly. Leaving children without vaccination out of laziness or saving vaccines is one of the most reported problems. Thus, Proper corrective measures must be taken immediately to check the quality of immunization coverage, performance of vaccinators otherwise the eradication program may be adversely affected.

\section{H. Inadequate management and Poor co-ordination}

A good management in any system led to high beyond expectations. Current vaccination system in Pakistan has so much lacking, inadequate management from the health officers, medical staff and government is also one of the obstacles. Vaccination system has no appropriate and proper management. Poor co-ordination between vaccinators and senior staff ruins the quality of campaign. Inadequate supervision with the poor accountability of the vaccination campaign is the factors of the failure of immunization system. Unfortunately, polio reported cases, highlighting the deficiencies and negligence of health management system.

All these problems are causing polio still prevailing in Pakistan. There is a need for a technology-based system that can compensate most of these problems identified during surveys. In this paper, we propose such a polio vaccination information system which is based on mobile technology and helps overcoming all challenges.

The figure 6 depicts the process adopted during the existing vaccination system.

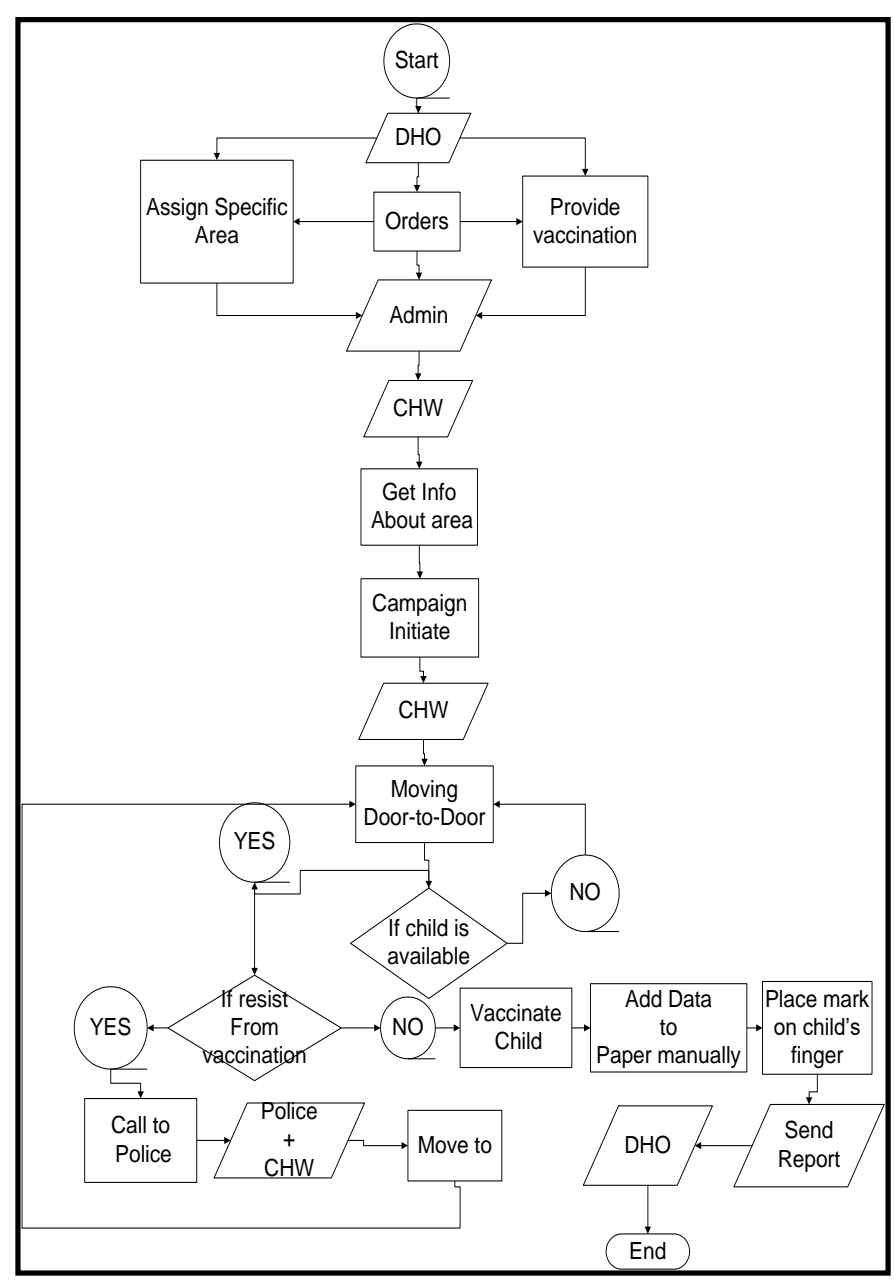

Fig. 6. Current Polio Vaccination System of Pakistan

In this model of current system, orders arrive from the District Health Quarter (DHQ) to the District Health Officer (DHO) about the campaign initiate. Then, orders along with schedule dates and vaccinations given to the administration of specific areas. Admin assign these to Community Health Workers (CHW) for the further vaccination process. $\mathrm{CHW}$ get information about their given areas then initiate campaign.

When campaign initiates, CHW move door-to-door, if child is available and at home then they are immunized by polio vaccine. Then $\mathrm{CHW}$ add trivial information of the vaccinated child on a sheet of paper (see figures 3 and 4) and put mark on child's finger. If someone shows resistance during immunization process like refusal of vaccine to their child then they simply inform the local police. This process of vaccination to the children will go on and health vaccinators visited house one by one in specific areas. During house-tohouse visit, if child is not present at home then vaccinators visit again and again to check the availability of the child for the vaccination. $\mathrm{CHW}$ mentioned the absence of child on the 
given sheet of paper, during campaign interval. When campaign time period comes to an end then report submitted to the DHO.

Above model illustrate this whole scenario of current polio vaccination system. Campaign process is so simple and doesn't have any strategic tactics or advanced technology. There is not any sort of electronic data involvement. Sheet of paper is being used to write the temporary data for an instance. System doesn't have any new or advanced technique for the missed children. Top most is the unsafe working environment for the vaccinator. There is no any adequate security given to the health provider.

\section{PROPOSED TECHNOLOGY ORIENTED PVS FOR PAKISTAN}

This model represents the proposed polio vaccination system. Model represents the current vaccination system with the real time technology, where GPS, remainder recall system, tracking system and monitoring system involved. Proposed system involved electronic data, and data will be updated correspondingly.

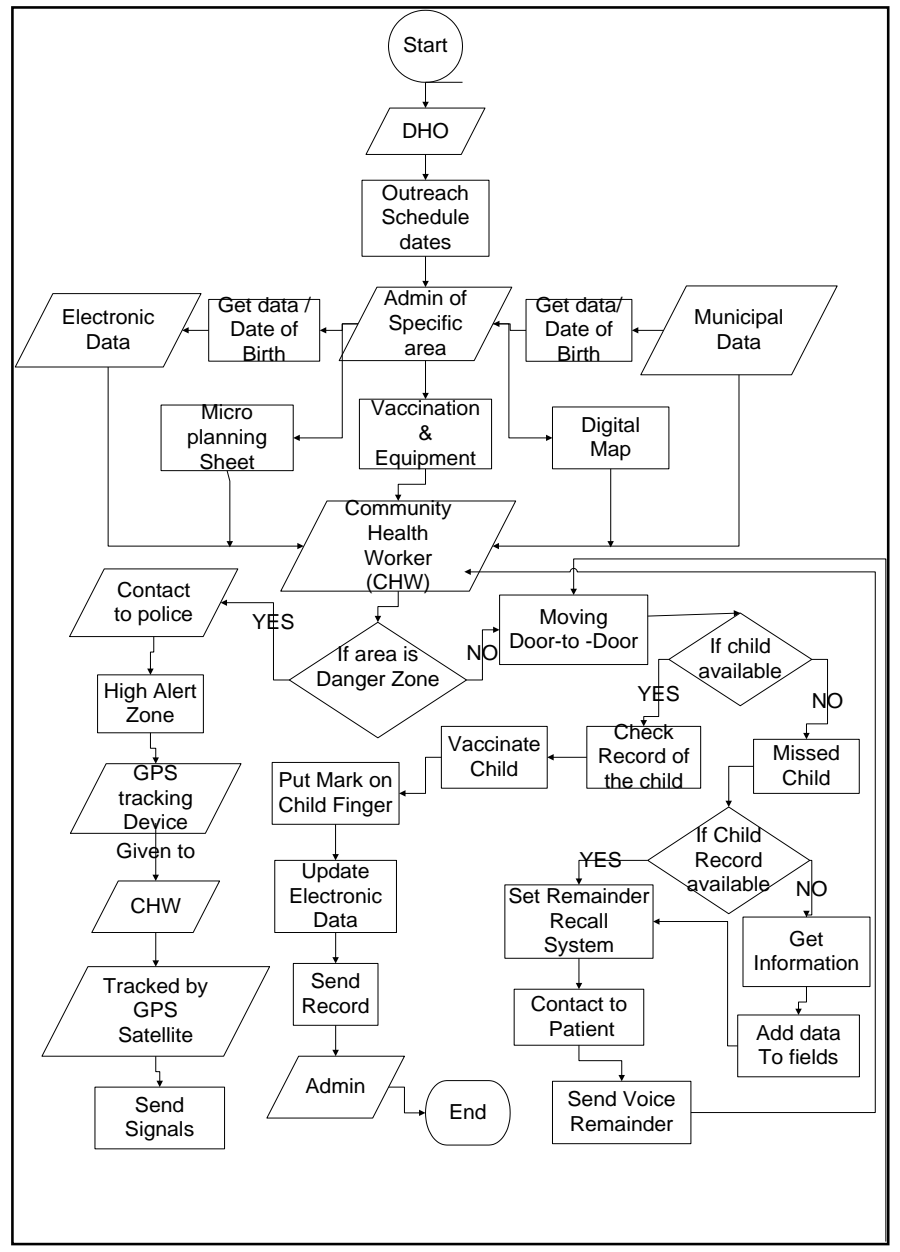

Fig. 7. (a) Proposed Scenario of Polio Vaccination System

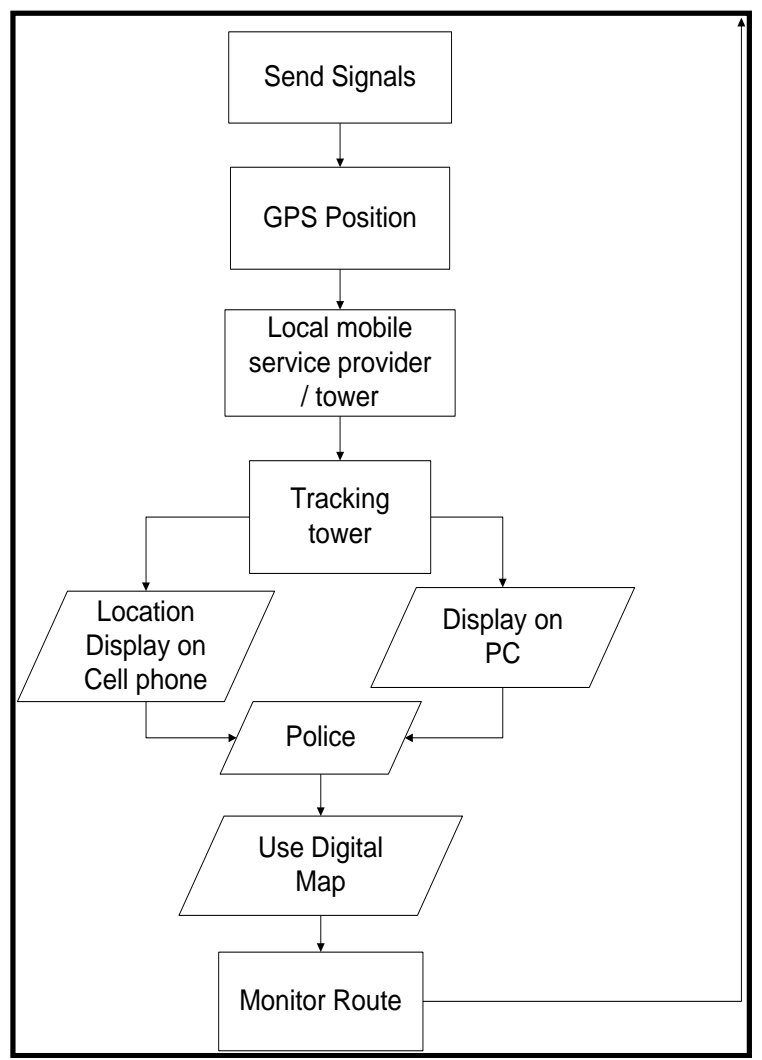

Fig. 7. (b) Proposed Scenario of Polio Vaccination System

The flow chart in figure 7-B represents the proposed polio vaccination system and its work flow. System starts with the schedule and orders from the DHO. Outreach program of immunization will initiate by administration according to the given dates. Administration of specific areas gets the data of infant from municipal. Now this data will be converted into electronic data for the further use and for the secure work. This electronic record along with its credentials given to the CHWs. Vaccination, micro planning and digital map will also be given the CHWs. Micro planning sheets consist of detail work flow and digital map of their specific areas for their convenience as well as to cover up the whole area sequentially. Thus, CHW will move door-to door by having all these possessions. Most of the polio affected areas of Pakistan are high risky, such areas should be heighted as a danger zone and need high precautions of safety. So, it's better to check whether area is lying in danger zone region or not. If area lies out of the danger region, means area is safe there is no need high safety precaution. For the safe areas, CHWs move door-to-door, if child is available then CHWs will vaccinate the children and update the electronic record against the child's data. This updated record will be shown to the admin as well for the further monitoring. This process will be going on. If area is in danger zone then, CHWs will go with police for the immunization. High alert will be at local police quarter and administration will also keep checking status of vaccination through vaccinators and police department. A 
tracking system will be given to the vaccinators and through tracking system, vaccinators performance will be measure and will be shown to admin as well as local police head quarter. Signals of the tracking system will be shown to the local PC or cell phone. If any mishap will occur or vaccinators need more security, then through tracking signals police force with move with the digital map as well. So they reached on time and get over the worst situation easily. If child is not available at home, then child will be included in the list of missed children electronically. CHWs will check whether record against missing child is available or not. If record will be provided then CHWs sent voice remainder to their corresponding numbers. Re-contact the guardian or the patient again for the immunization. If no data will be present then, administration will get the information against the patient.

\section{A. Re-Campaign Model of Proposed Polio vaccination system in Pakistan}

In re-campaign polio vaccination system, re-campaign for the missed children are as important as other processes to be accomplished. High rate of missed children is one of the main reasons of having polio virus in Pakistan. Thus to get over it, an accurate and adequate re-campaign is the need of time. In re-campaign process, $\mathrm{CHWs}$ get the data of missed children from the electronic record. CHWs check the area during doorto-door vaccination process, whether more missed children is in remote areas or the city area. Then CHWs will move after getting the required information of the given area so they will perform better.

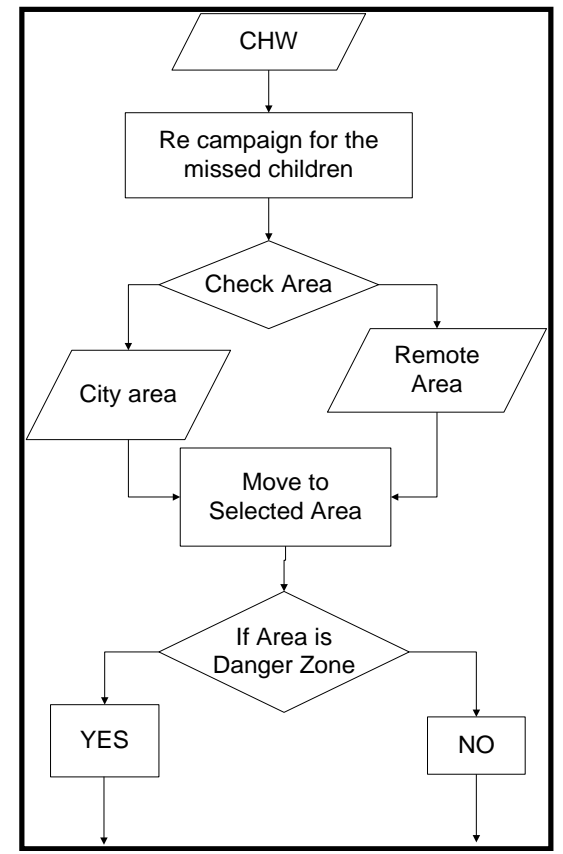

Fig. 8. Proposed Re-Campaign Scenario of Polio vaccination system

\section{FORMAL MODELING OF PROPOSED PVS}

\section{A. Formal Verification}

Formal verification efforts to give an answer for Functional check that sidesteps these issues by confirming (or refuting) accuracy of the configuration concerning the particular. A formal confirmation of a property is proportional to thorough testing of the configuration concerning that property [14].

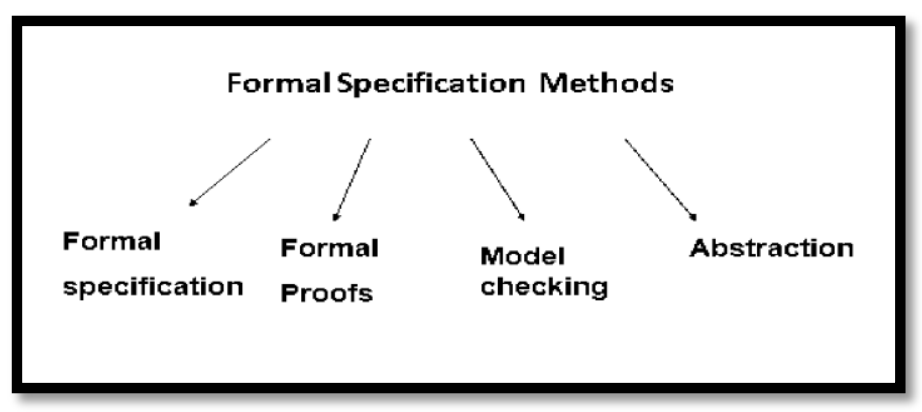

Fig. 9. Main Division of Formal Methods

\section{Model Checking}

Model checking has many kinds of displaying formalisms like Colored Petri Nets (CPNs) graphical demonstrating dialect. A CPN model is a dialect for displaying and acceptance of simultaneous and conveyed frameworks and different frameworks in which concurrency, synchronization, and correspondence assumes a noteworthy part [15, 16, 17].

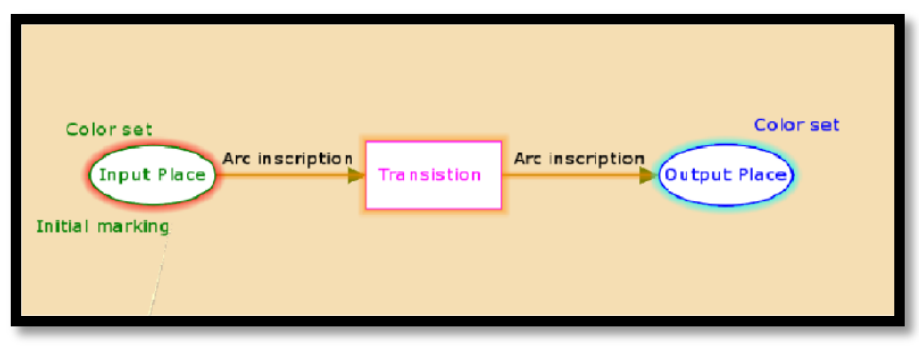

Fig. 10. Structure of Colored Petri Nets

Colored Petri Nets, where data is joined to every token. The data can be examined and changed when a move fires. For most applications, this speculation of customary Colored Petri Nets permits the client to make more sensible depictions, because of the way that equivalent sub Nets can be collapsed into one another, yielding a much littler Net. For reasons unknown spot invariants and achieve capacity trees, noteworthy techniques for standard CPNs, can be summed up to pertain for Colored Petri Nets [18].Colored Petri Nets and Predicate/transition-Nets are very intently linked, in the way that Colored Petri Nets have been established as an alteration of Predicate/transition-Nets, in order to avoid some practical troubles which rise when the technique of place-invariants is general to apply for Predicate [18].Colored Petri Net concept delivers potent analysis techniques used to verify the accuracy of workflow procedures. Acceptance of a model-driven approach, joined through comprehensive verification methods can make available a key solution for making qualitative and quantitative calculations about the possible system behaviors $[18,19]$.

Our work details a study in Verification of a system using Formal Modeling of Polio Vaccination System Using Colored Petri Nets. We show how support for features of correctness and verification in Colored Petri Nets enables demonstration $[19,20]$. 
System verification is a central part in the software development process. It requires however, the system under the test of Colored Petri Nets to be at least moderately implemented. Also the practical verification of the systems working is exposed using simulations. Based on the usage of Colored Petri Nets as a specification tool we present an approach allowing the application of systems processes and transitions. As an additional benefit, the well-defined semantics of Colored Petri Nets enforces completeness and consistency of the system specification. The execution of the described technique is relied on the development of the vaccination system [21, 22]

\section{FUNCTIONAL ARCHITECTURE}

Colored Petri Nets models are well structured and provide planned modeling. Interactive simulations are created in using CPN tool. First we plot an idea to model the system using formal methods of software engineering [23, 24].

In Colored Petri Nets the conditions are depicted as a number of tokens presented as places and graphically represented in ellipse symbol. The data values are of different types like String, Integers, Unit, Boolean. We can combine some values with arithmetic operators. The actions are triggered in form of Transitions. In Colored Petri Nets that are designed the data flows through Arcs. The Arcs gets the Input from the tokens and process them in Transition as an Output. This model gives a complete strength on the conditions. For example, if we give a valid argument then we gets the desired result, otherwise the token will not flow. Green highlighted of transition shows that transition is active and flow work correctly $[25,26]$.

\section{Area Zone Scenario}

In figure 11-A, CPN of our system shows the basis scenario of area zone. DHO and Admin assign the basic essentials to the field worker to initiate the campaign. The new constrain used between $\mathrm{CHW}$ and check area is "new precedence constrain". This constrain will shows the precedence between transitions [27, 28]. Then field worker first check the area, whether health care provider move to safe area zone or danger region. In this colored Petri Net it is just start of simulation, when all tokens are ready to move by simulation with their prescribed variables and values [29, 30].

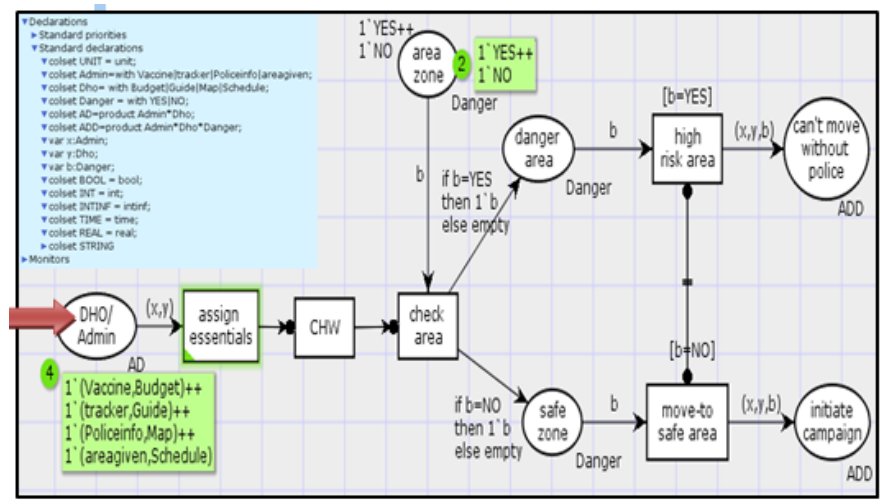

Fig. 11. (a) Main Simulation for the Area Zone Scenario
In figure 11-B, we are illustrating the variable declaration of our Proposed Polio Vaccination System. Colset represents the declaration with it specific value and each colset have some variable. Each variable belongs to its corresponding colset.

In second process CHW will select one area, either safe or danger area, so we use "new not co-existence constraint". This constraint show that both transitions will not enable at the same time, only transition will exist at once. It shows that CHW will move either towards safe area or danger area.

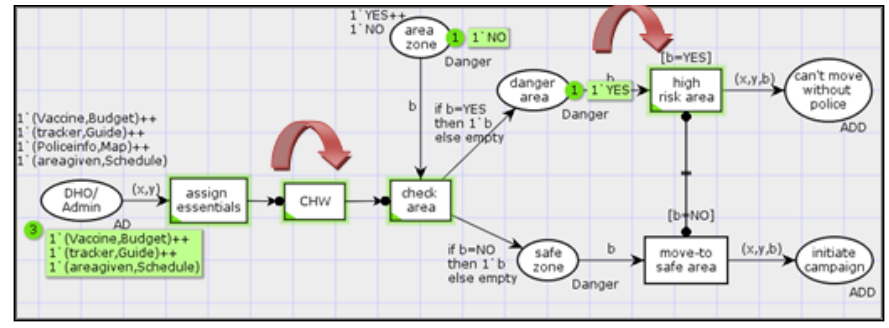

Fig. 11. (b) High risk area enables all transition

It shows that CHW will go to the danger area and only high risk area transition is enabled and all tokens passed and green highlighted the transition. Here value of variable " $b=$ YES" depict that area is danger and CHW could not move without police. Output will be shown in the figure mentioned below.

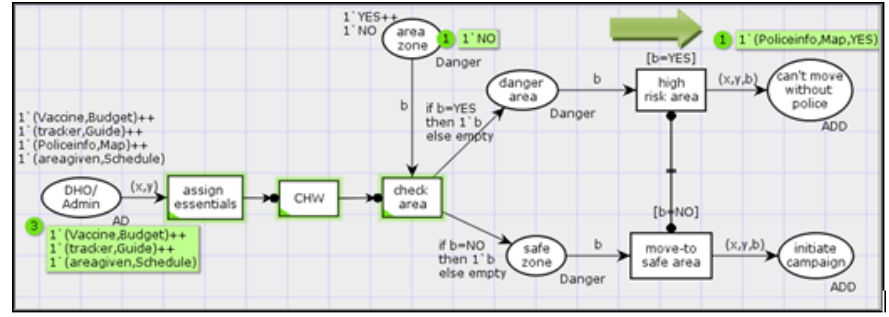

Fig. 11. (c) All token of High risk flow and shown in Output

In the above figure 11-C, output will be shown on the place. Where all token flows perfectly and depict that area is in danger zone and CHW can't move without police for the vaccination is shown with the green arrow.

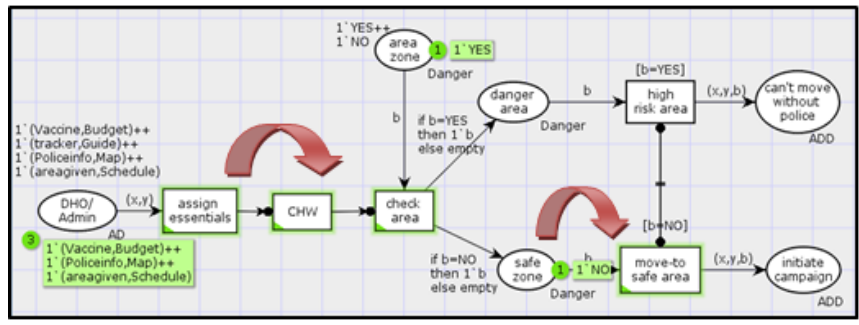

Fig. 11. (d) Safe area token followed and enabled all transition

In this CPN figure 11-D, it shows that CHW will go to the safe area and all the transition is enabled of the safer zone area, whereas " $\mathrm{b}=\mathrm{NO}$ " represents that there is no danger, CHW can move in safe area zone and flow of tokens will be shown with red arrows in figure 11-E. 


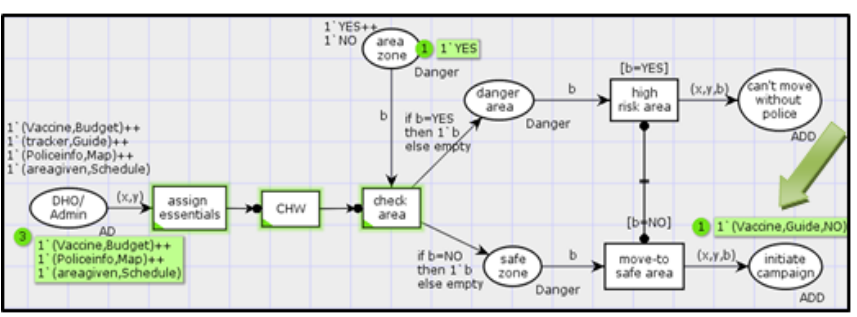

Fig. 11. (e) All tokens of Safe area flow and shown in Output

\section{Safe Zone Scenario}

In safe zone scenario of figure 12-A, this is the initial state of safe zone area, where no tokens are flowing. CHW will check if there is no danger then $\mathrm{CHW}$ will move, else tokens will not flow. Different variable are used in this scenario, "b" is the variable used to declare the danger, " $x$, $y$ " variables are for the DHO and Admin, which will assign to the CHW to move door-to-door. In this Petri Net it is just start of simulation, when all tokens are ready to move by simulation with their prescribed variables and values.

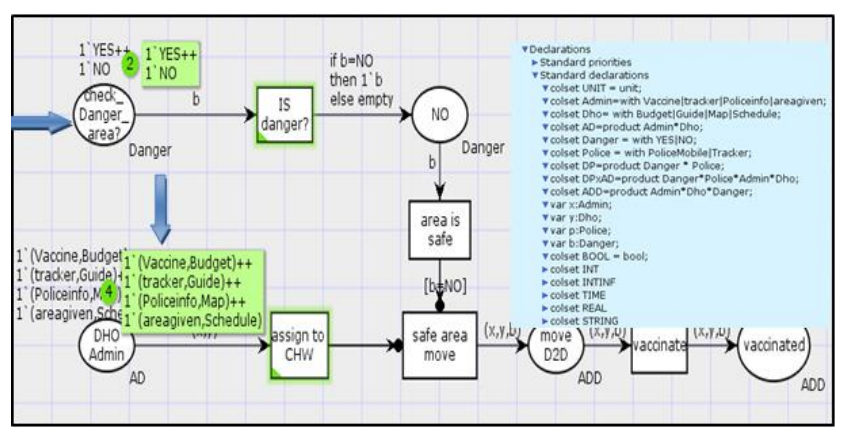

Fig. 12. (a) Main Simulation for the Safe Area Zone Scenario

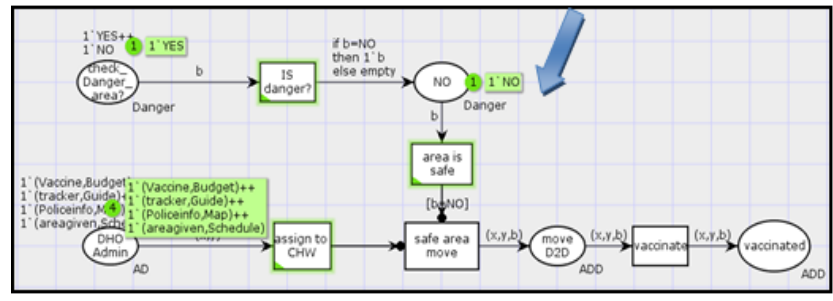

Fig. 12. (b) Simulation for No danger Zone Scenario

In above $\mathrm{CPN}$ figure 12-B, there is no danger the transition labeled with "area is safe" is highlighted and shows that token flowing accurately. If danger means " $b=$ YES" then no token will flow and it will empty. "New Precedence constraint" is between transition shows the precedence level.

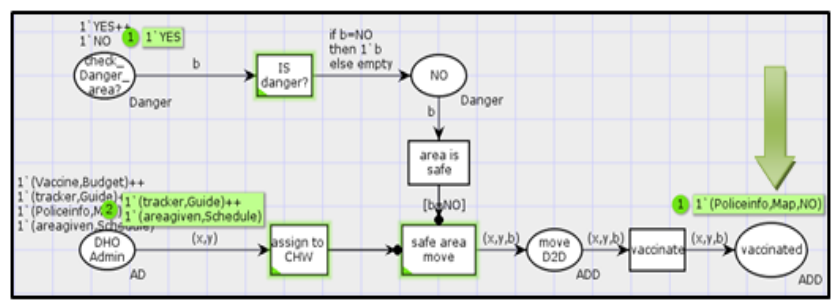

Fig. 12. (c) All tokens of Safe area flow and shown in Output
In this Figure 12-C, variable " $b=N O$ ", means safe area, so transition labeled with safe area is highlighted. All the tokens are flowing accurately and transition is enabled. Output will be shown in the last place.

\section{Danger Zone Scenario}

The first CPN figure 13-A represents the simulation of danger area and shows the basis scenario of danger area zone. There are more than one initial states of this CPN, first one is to check out that assign area is lies in danger zone or not. Second initial state is DHO and Admin assign the basic essentials to the field worker to initiate the campaign. The new constrain used around the transition labeled with "Danger move high alert" is "new precedence constrain". This constrain will shows the precedence between transitions. Then field worker first check the area, whether health care provider move to danger region and are high alert. In this Petri Net it is just start of simulation, when all tokens are ready to move by simulation with their prescribed variables and values.

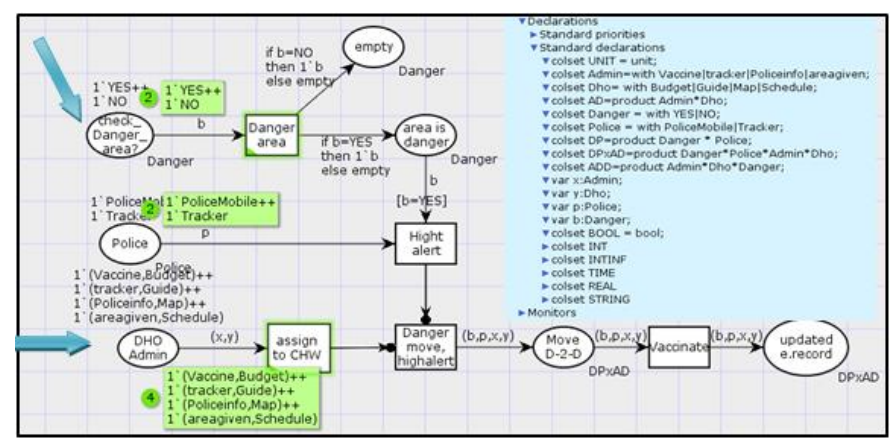

Fig. 13. (a) Main Simulation for the Danger Area Zone

This CPN model 13-A depicts the most of the functionality of our danger zone area, here are initial transitions represent danger area and $\mathrm{CHW}$ need police to go for the vaccination. It shows all tokens are in their initial states and ready to simulate. It shows all tokens are in their initial states and ready to simulate.

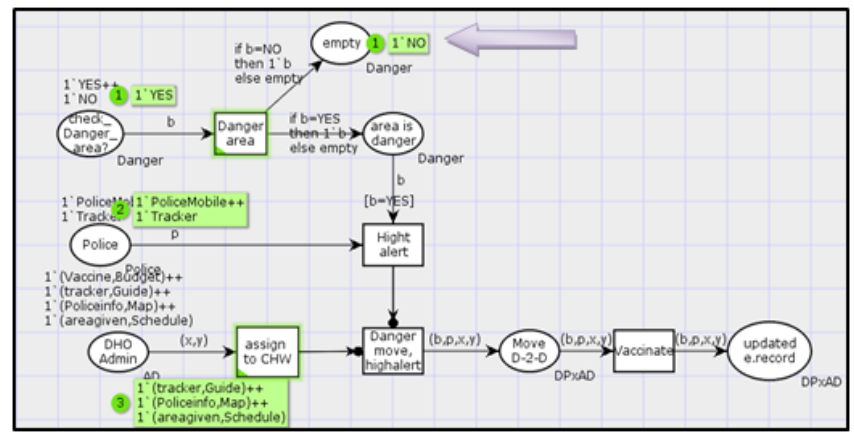

Fig. 13. (b) Simulation showing output with no danger in area

This CPN 13-B, initiate with the labeled "check danger area", CHW will check the area first to move for vaccination. This Figure 13-B, depict that if area is no danger, then it shows the empty place means no further transition or place going to be held. Tokens are flowing accurately and output shown on the place labeled with "empty". 


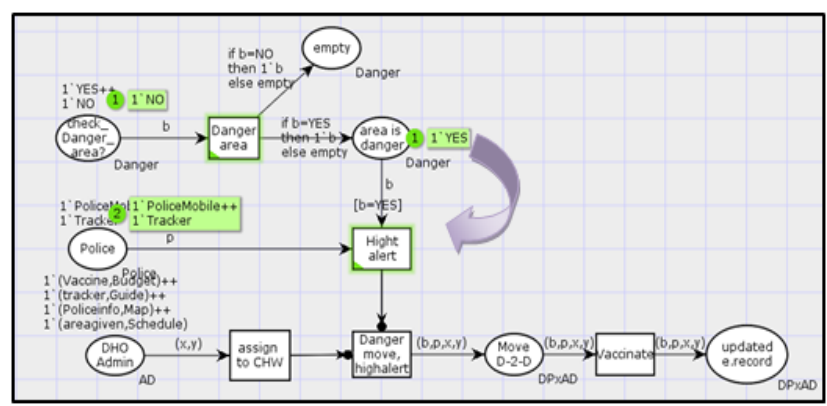

Fig. 13. (c) Simulation of High alert in danger area

If area will be in danger zone than high alert will green and enabled. In figure 13-C, shows danger area transition is shown highlighted means area is danger so police will interact with the health provider and high alert will be enabled.

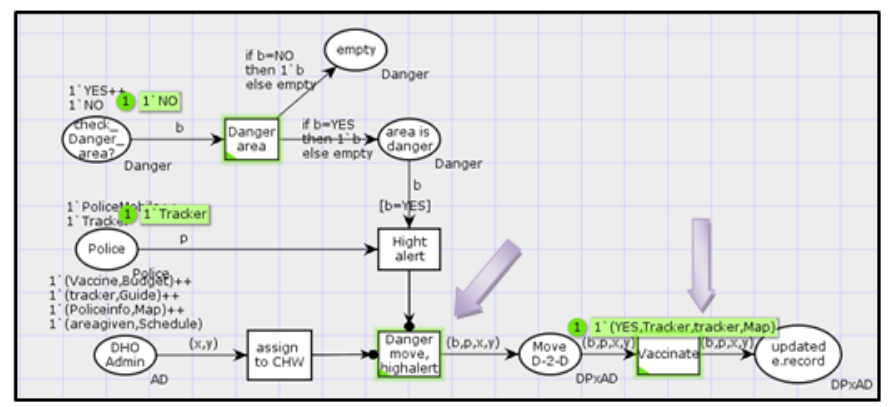

Fig. 13. (d) Simulation of move in danger area

Figure 13-D, shows all token flows perfectly and depict that area is in danger zone and high alerted. CHW move with police for the vaccination along with all essentials.

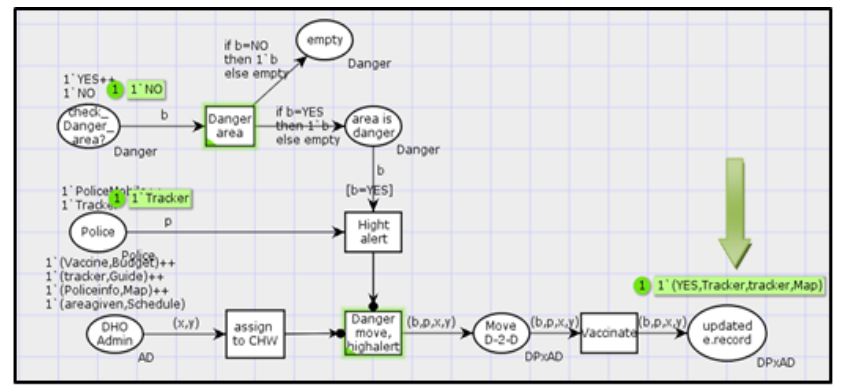

Fig. 13. (e) All token of Safe area flow and shown in Output

Final simulation of CPN 13-E is Showing the flow of all token from danger area if it will be "YES" and it highlights the "danger move, high alert" and token are functioning accurately and Petri Nets working perfectly. Green arrow shows the simulation of whole CPN module.

\section{Child Availability Scenario}

This CPN represents the scenario of availability of child. CHW will check the whether the child is available or not. If not available then child be in missed list, if child will be present at home for the vaccination then $\mathrm{CHW}$ will move further with the essentials. In this Petri Net it is just start of simulation, when all tokens are ready to move by simulation with their prescribed variables and values CPN shown with the red arrows in figures below.

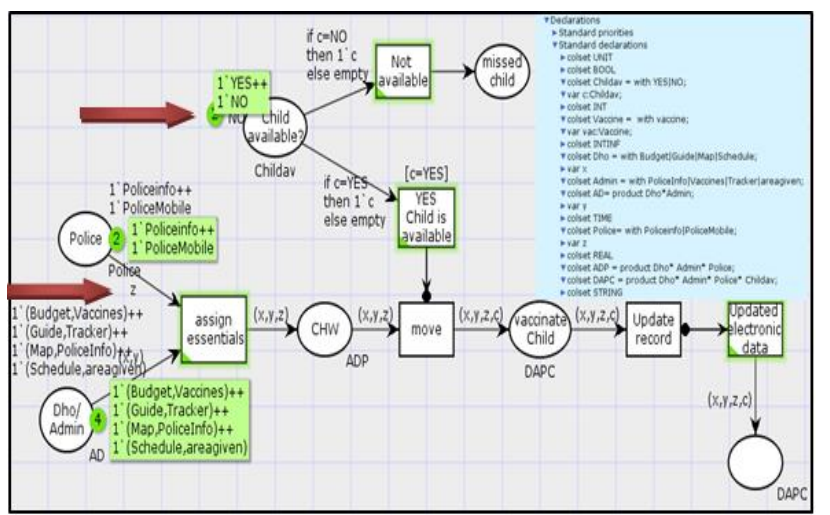

Fig. 14. (a) Main Simulation for the Child Availability Scenario

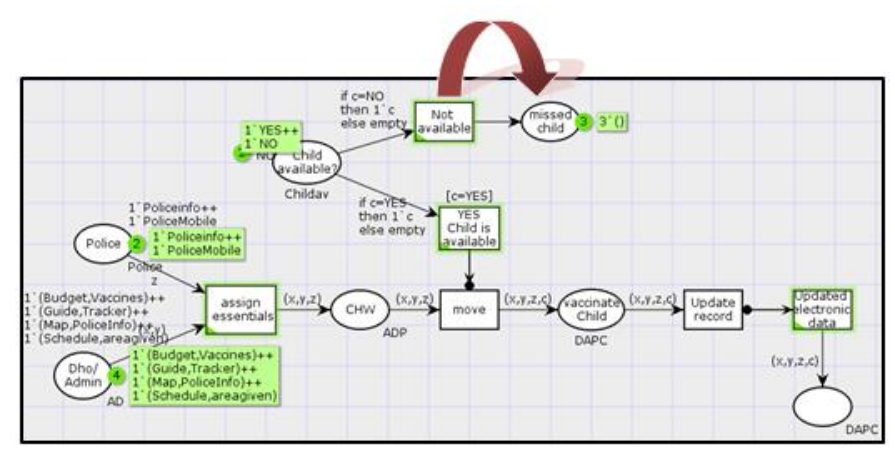

Fig. 14. (b) Simulation of missed child

In above $\mathrm{CPN}$, it is shown that first tokens flowing to check the availability of child. Child is not available and transition is enabled labeled with "Not Available" and output shown in place of missed child.

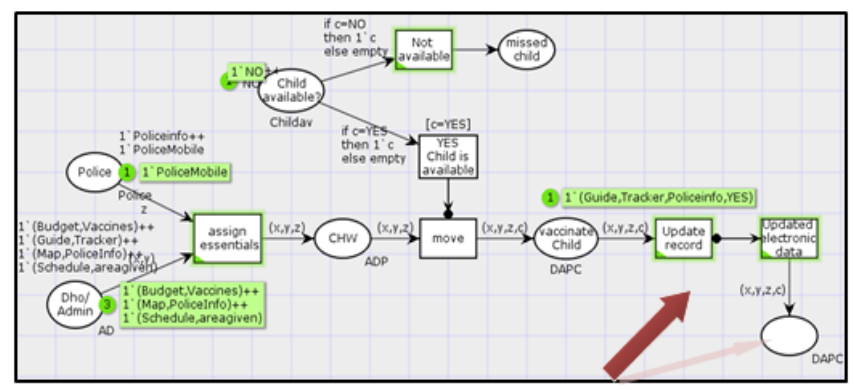

Fig. 14. (c) All token of Safe area flow and shown in Output

Figure 14-C shows simulation of vaccination child if available is exposed; transition is enabled shown with the green highlights. Token flowed accurately and Colored Petri Nets working correctly.

\section{Missed Child Scenario}

In this Petri Net it is just start of simulation, when all tokens are ready to move by simulation with their prescribed variables In this Missed Child CPN, CHW will check the whether the child is missed or not. If missed then child be in missed list, if child will not be present at home for the vaccination then CHW will move further and contact its relevant information for the vaccination and then update the record. In this scenario, there is new constrain between 
children available or not is "new not co-existence constraints" means transition both will not happened at the same time. If child will not be present then move to another transition and if not missed then move with different transition as shown in figures 15-A.

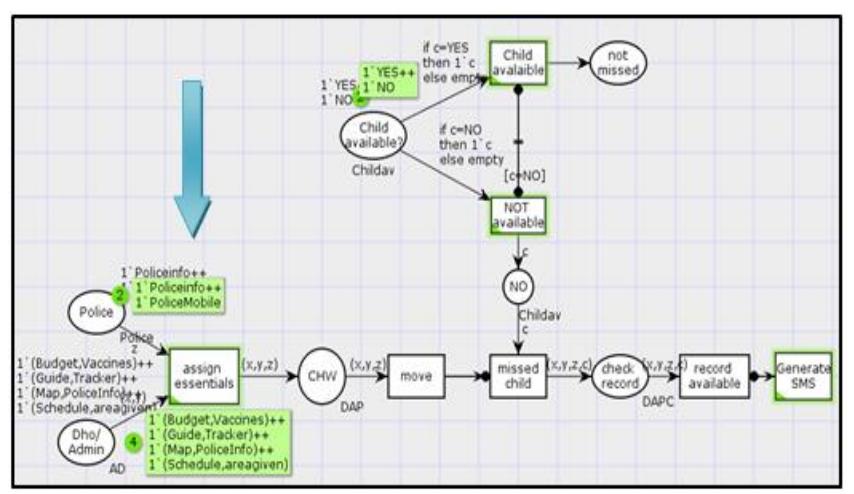

Fig. 15. (a) Main Simulation for the Missed Child Scenario

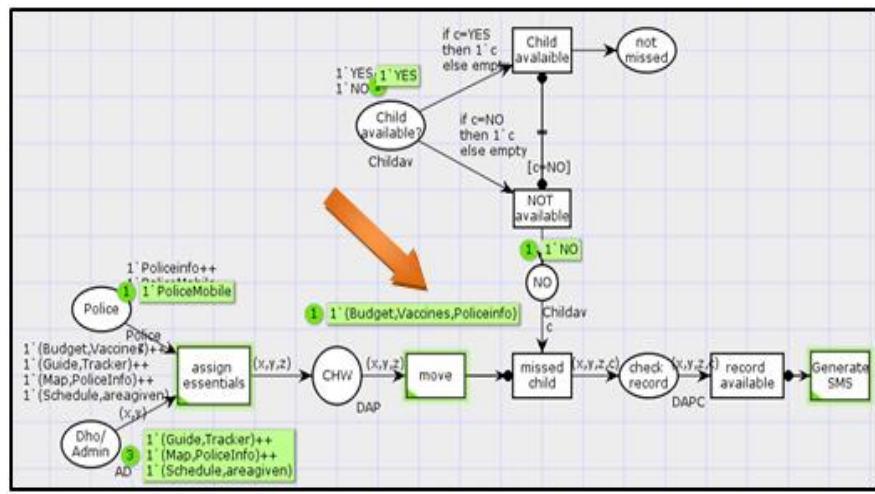

Fig. 15. (b) Simulation for the Missed Child with followed token

This figure 15-B shows that $\mathrm{CHW}$ will move with the essentials and check the presence of child first. If child is not available then "No" label and token flowed.

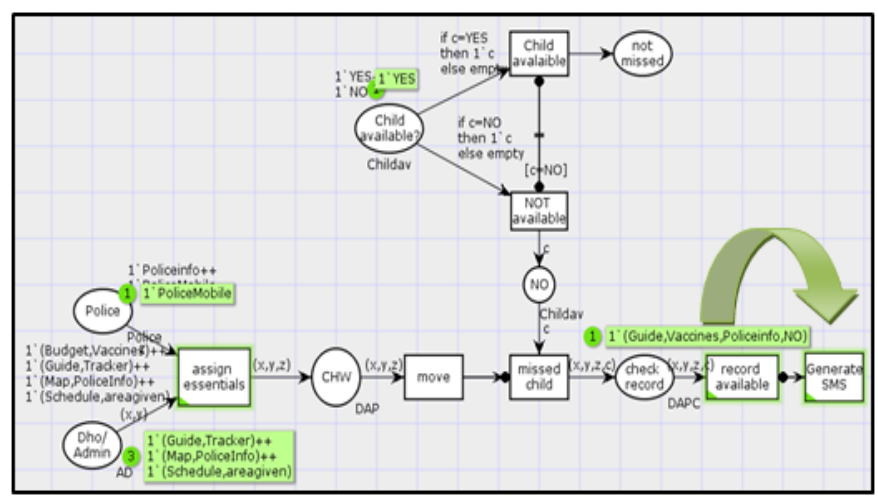

Fig. 15. (c) Simulation shown flow of token along with output

Figure 15-C shows simulation depicts the flow of tokens of missed child scenario and describes the most of the functionality of missed child picture. It shows all tokens are in their final states.

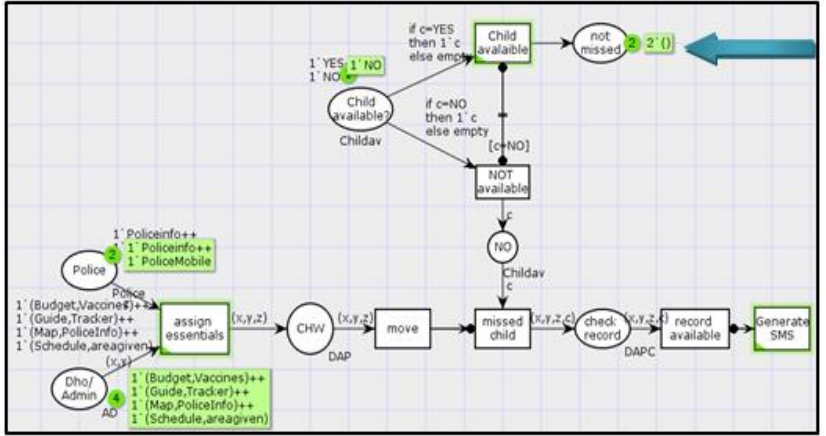

Fig. 15. (d) Simulation of the Not Missed Child

This CPN model 15-D, depicts that if child will be present at home then child will not be included in missed list, and transition is enabled and output is shown in the last output place.

\section{Re-campaign Scenario}

Colored Petri Nets based models are designed in many forms for research and formal modeling of different case studies Therefore, performance using Colored Petri Nets must rely on different simulations to show the complete performance measures for a supposed system.

In re-campaign $\mathrm{CPN}, \mathrm{CHW}$ initiate the campaign with the missed children only. $\mathrm{CHW}$ initiate the campaign with all of its essentials and move to check the area if the given area is reside in city or it will be rural area. All tokens are ready to move by simulation with their prescribed variables and values, variables are initialized to accomplish the process for missed child and CPN shown in figure 16-A.

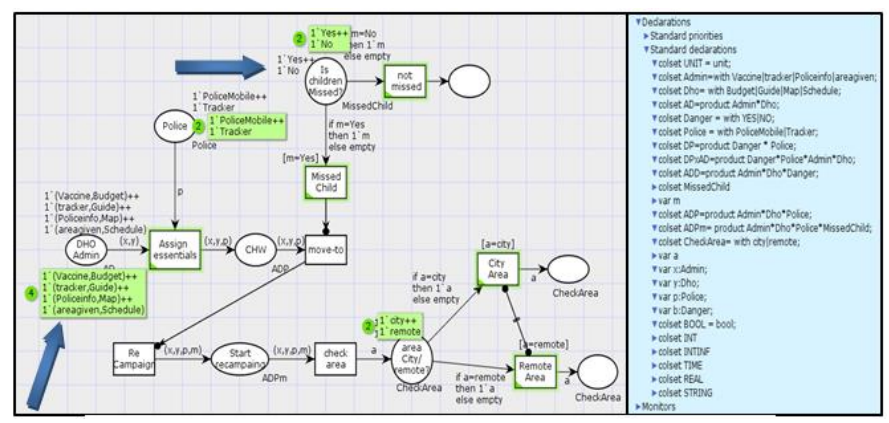

Fig. 16. (a) Initial Simulation of the Not Missed Child

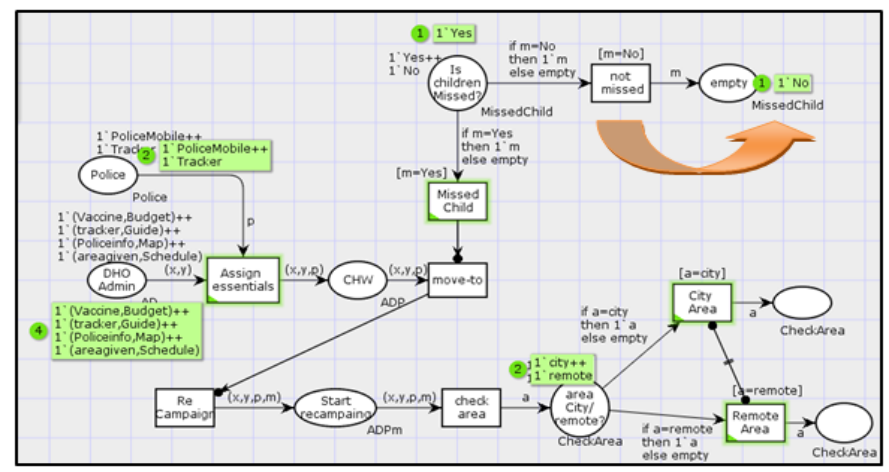

Fig. 16. (b) Simulation of the Not Missed Child 
In this CPN 16-B, it shows the simulation of re-campaign It shows whether child is missed or not, if not missed then it will be empty places. Tokens are flowing accurately and Colored Petri Nets are working correctly, shows with the pointed arrow.

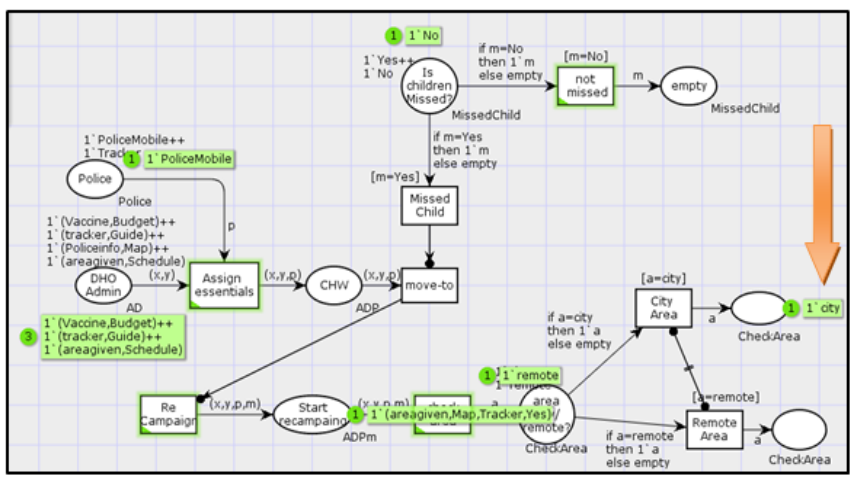

Fig. 16. (c) Simulation of re-campaign of city area

In this figure 16-C, shows the simulation of re-campaign of city area. This CPN model represents the flow of token and output represents with the sign of arrow.

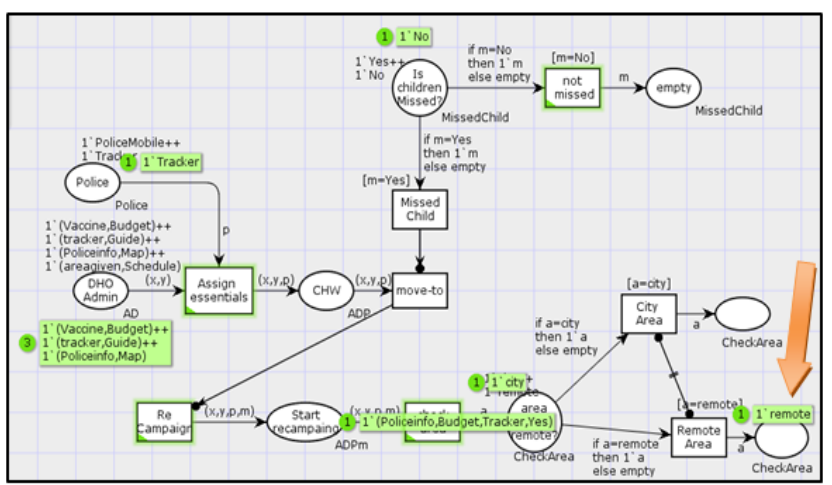

Fig. 16. (d) Simulation of re-campaign with remote area

Final simulation of this CPN 16-D, showing all the data is available in the form of output and remote area will be selected. This model is representing the flow of tokens with sign of arrow and transition is enabled.

\section{CONCLUSIONS}

After scrutinized the existing polio vaccination system, we proposed technology oriented secure polio vaccination system. Our purpose is to build a system which makes the polio vaccination system effective and competent so we will be able to stand against the wild polio virus and make the Pakistan free from polio virus. The focus of this paper is directed towards the use of advanced tools in current vaccination system. The goal of the work offered is to commence a technology to make the polio vaccination system efficient in general and security perspective. This thesis investigate those tools which can be used to make a vaccination system effective and secure for the vaccinators which got death threat and find them defenseless in the fulfillment of their duties during vaccination campaign. Without proper security of health workers and general public it is not possible to carry out a successful campaign. On the other hand ourselves).by making Polio vaccination system's model we research a framework before we build it and to check the correctness of our proposed system by formal modeling. We also talked about the use of Colored Petri Nets to the Polio Vaccination system ends up being an application area which could profit by the elements of Colored Petri Nets. There are numerous great explanations behind utilizing Colored Petri Nets for data displaying and examination. CPN model is a depiction of displayed framework, and it could be utilized as an important feature (of a framework which we need to assemble) or as a presentation (of a framework which we need to disclose to other individuals. We did formal modeling of our Proposed technology oriented secure Polio Vaccination System. We verified our proposed model and by using Colored Petri Nets stimulate the different scenarios of our system. Hence we proved the correctness of our system.

\section{REFERENCES}

[1] Kazi, Adbul Momin, M. Khalid, and A. N. Kazi. "Failure of polio eradication from Pakistan: Threat to world health." J Pioneer Med Sci 4.1 (2014): 8-9.

[2] GIS Mapping \& GPS Tracking for Polio in Nigeria. Available from: http://mobile.thegatesnotes.com/Topics/Health/GIS-Mapping-GPSTracking-for-Polio-in-Nigeria

[3] Stockwell, Melissa S., and Alexander G. Fiks. "Utilizing health information technology to improve vaccine communication and coverage." Human vaccines \& immunotherapeutics 9.8 (2013): 18021811.

[4] Shah, Syed Zawar, et al. "WHY WE COULD NOT ERADICATE POLIO FROM PAKISTAN AND HOW CAN WE?." Journal of Ayub Medical College Abbottabad 28.2 (2016): 423-425.

[5] Dutta, Anil. "Epidemiology of poliomyelitis—options and update." Vaccine 26.45 (2008): 5767-5773.

[6] Kay, Misha, Jonathan Santos, and Marina Takane. "mHealth: New horizons for health through mobile technologies." World Health Organization 64.7 (2011): 66-71.

[7] Bhaumik, Soumyadeep. "Polio eradication: Current status and challenges." Journal of family medicine and primary care 1.2 (2012): 84.

[8] Roush, Sandra W., Trudy V. Murphy, and Vaccine-Preventable Disease Table Working Group. "Historical comparisons of morbidity andmortality for vaccine-preventable diseases in the United States." Jama 298.18 (2007): 2155-2163.

[9] Barau, Inuwa, et al. "Improving polio vaccination coverage in Nigeria through the use of geographic information system technology." Journal of Infectious Diseases 210.suppl 1 (2014): S102-S110.

[10] Mehmood, Khalid, et al. "POLIO VACCINATION."

[11] Bhaumik, Soumyadeep. "Polio eradication: Current status and challenges." Journal of family medicine and primary care 1.2 (2012): 84.

[12] Kazi, A. M., et al. "Monitoring polio supplementary immunization activities using an automated short text messaging system in Karachi, Pakistan." Bulletin of the World Health Organization 92.3 (2014): 220225.

[13] http://www.healthline.com/health/poliomyelitis.

[14] Parunak, H. Van Dyke. "Visualizing agent conversations: Using enhanced dooley graphs for agent design and analysis." Proceedings of the second international conference on multi-agent systems (ICMAS'96). 1996.

[15] Clarke, Edmund M., and E. Allen Emerson. "Design and synthesis of synchronization skeletons using branching time temporal logic." Workshop on Logic of Programs. Springer Berlin Heidelberg, 1981.

[16] Jensen, Kurt, and Lars M. Kristensen. Coloured Petri nets: modelling and validation of concurrent systems. Springer Science \& Business Media, 2009

[17] Brauer, Wilfried, Wolfgang Reisig, and Grzegorz Rozenberg, eds. Petri Nets: Central Models and Their Properties: Advances in Petri Nets 1986, 
Part I Proceedings of an Advanced Course Bad Honnef, 8.-19. September 1986. Vol. 254. Springer, 2006.

[18] Emerson, E. Allen, and Jai Srinivasan. "Branching time temporal logic." Workshop/School/Symposium of the REX Project (Research and Education in Concurrent Systems). Springer Berlin Heidelberg, 1988.

[19] Nodine, Marian H., and Amy Unruh. "Facilitating open communication in agent systems: The infosleuth infrastructure." International Workshop on Agent Theories, Architectures, and Languages. Springer Berlin Heidelberg, 1997.

[20] Jensen, Kurt. Coloured Petri nets: basic concepts, analysis methods and practical use. Vol. 1. Springer Science \& Business Media, 2013.

[21] L. Wells, S. Christensen, L. M. Kristensen, and K. Mortensen Simulation based performance analysis of web servers. In R. German and B. Haverkort, editors, Proceedings of the 9th International Workshop on Petri Nets and Performance Models, pages 59-68. IEEE, 2001.

[22] B.Lindstrom and L. Wells. Annotating coloured Petri nets. To appear in the proceedings of the Fourth Workshop and Tutorial on Practical Use of Coloured Petri Nets and the CPN Tools (CPN'02), 2002.

[23] Christensen, S., Kristensen, L.M.: State Space Analysis of Hierarchical Coloured Petri Nets. In: Farwer, B., Moldt, D., Stehr, M.-O. (eds.):
Proceedings of Workshop on Petri Nets in System Engineering Modelling, Verification, and Validation. Department of Computer Science, University of Hamburg, 1997, pp. 32\{43, Report no. 20.

[24] Kristensen, Lars M., Soren Christensen, and Kurt Jensen. "The practitioner's guide to coloured Petri nets." International Journal on Software Tools for Technology Transfer (STTT) 2.2 (1998): 98-132.

[25] Gordon, Steven Donald. "Verification of the WAP transaction layer using coloured Petri nets." (2001).

[26] Jensen, Kurt. "Coloured Petri nets: A high level language for system design and analysis." International Conference on Application and Theory of Petri Nets. Springer Berlin Heidelberg, 1989.

[27] Zimmermann, Armin. "Colored petri nets." Stochastic Discrete Event Systems: Modeling, Evaluation, Applications (2008): 99-124.

[28] Cost, R. Scott, et al. "Modeling agent conversations with colored petri nets." Working Notes of the Workshop on Specifying and Implementing Conversation Policies. 1999

[29] Liu, Dongsheng, et al. "Modeling workflow processes with colored Petri nets." computers in industry 49.3 (2002): 267-281.

[30] Cost, R. Scott, et al. "Using colored petri nets for conversation modeling." Issues in Agent Communication. Springer Berlin Heidelberg, 2000. 178-192 